\title{
Editorial
}

Liebe Leser_innen,

kurz vor Jahresende erscheint nun die zweite Ausgabe von s u b $\backslash \mathbf{u} \mathbf{r} \mathbf{b}$ a $\mathbf{n}$. In der Zeit zwischen dem ersten und zweiten Heft ist einiges passiert, von dem wir hier kurz berichten möchten. Zunächst bedanken wir uns für die vielen positiven Reaktionen, die uns nach der Veröffentlichung des ersten Heftes erreichten. Das hat uns gefreut und motiviert, mit dem Projekt fortzufahren. Gefeiert haben wir mit einigen Autor_innen und Unterstützer_innen im Berliner Südblock am Kottbusser Tor im Rahmen der RC-21-Konferenz. Ein weiteres Ereignis ist der Geographentag im Oktober in Passau gewesen, auf dem wir die Gelegenheit hatten sub\urban vorzustellen. Gefreut haben wir uns auch über die mehrfach herausgestellte positive Wahrnehmung des Debattenteils, den wir in der ersten Ausgabe zu Texten von Margit Mayer sowie Hartmut Häußermann/Walter Siebel eröffnet haben.

Positives berichten können wir zudem über die dringend benötigte finanzielle Unterstützung bei der Produktion von $\mathrm{s} \mathbf{u} \mathbf{b} \backslash \mathbf{u} \mathbf{r} \mathbf{b} \mathbf{a} \mathbf{n}$. Das vorliegende Heft wird von der Rosa-Luxemburg-Stiftung gefördert. Im Oktober haben wir einen Bewilligungsbescheid von der Deutschen Forschungsgemeinschaft (DFG) zu einer Anschubförderung für wissenschaftliche Zeitschriften erhalten. Somit werden wir in den kommenden drei Jahren in der Lage sein, zumindest einen Teil unserer Herstellungsvorgänge nach außen zu vergeben und uns stärker auf die redaktionelle Arbeit konzentrieren zu können. Außerdem freuen wir uns sehr über die Spenden von Privatpersonen und Institutionen im Rahmen von Förderabonnements. Diese tragen wesentlich zur Kontinuität der Zeitschrift bei. Schließlich gab es auch Fortschritte bezüglich einer weiteren materiellen Grundlage: Im November 2013 haben wir den Verein $\mathbf{s} \mathbf{u} \mathbf{b} \backslash \mathbf{u} \mathbf{r} \mathbf{b}$ a $\mathbf{n}$ e. V. gegründet, der von nun an der formale Herausgeber unserer Zeitschrift für kritische Stadtforschung ist.

Die zweite Ausgabe von $\mathbf{s} \mathbf{u} \mathbf{b} \backslash \mathbf{u} \mathbf{r} \mathbf{b}$ a $\mathbf{n}$ erscheint - herausgegeben von unseren Redaktionsmitgliedern Boris Michel und Nikolai Roskamm - als 
Themenheft, das sich zum Ziel gesetzt hat, die Debatten zur ,neoliberalen Stadt' und zur ,postpolitischen Stadt' zusammen zu bringen.

\section{Zu den Beiträgen in diesem Heft}

In ihrer Einleitung stellen Boris Michel und Nikolai Roskamm in einer ideengeschichtlichen Skizze heraus, dass die ,Postpolitikthese“ dem in der kritischen Stadtforschung etablierten Analyserahmen der ,neoliberalen Stadt' nicht entgegengesetzt ist, sondern vielmehr eine Erweiterung anbieten möchte. Die Rückverfolgung des Arguments von der Postpolitik führt zunächst zu den Arbeiten von Chantal Mouffe, Slavoj Žižek und Jacques Rancière und damit zu Vertretern der ,neuen Theorien des Politischen“. Die Privilegierung des Politischen, das hier als eine zusammenhaltende Klammer gedacht werden kann, lässt sich wiederum zur Konflikttheorie eines Carl Schmitts und zur Kontingenztheorie von Hannah Arendt zurückverfolgen. Dort angekommen eröffnet sich zwischen den beiden Thesen von der postpolitischen und der neoliberalen Stadt dann allerdings doch ein potentieller Dissens: Während Konflikt als Erklärungsmodell für die kritische Stadtforschung ebenso plausibel wie verbreitet ist, ist dort die mit dem Begriff des Postpolitischen ebenfalls transportierte Kontingenzthese weit weniger fest verankert (und auch weit weniger beliebt). In diesem Spannungsfeld verorten die beiden Herausgeber die in ihrem Themenheft versammelten Aufsätze.

Im Beitrag „Der Lärm des Politischen“ fragen Iris Dzudzek und Michael Müller, inwiefern sich in den Londonern riots aus dem Jahre 2011 eine Dimension des Politischen und politischer Subjektivität zeigt, die mit vertrauten sozialwissenschaftlichen und repräsentationspolitischen Deutungsmustern nicht zu fassen ist. Die Autor_innen untersuchen die in der medialen Berichterstattung und in den politischen Bewertungen verwendeten Rhetoriken und kontrastieren sie mit Stimmen der Revoltierenden, mit Daten zur sozialen Lage in London und mit dem radikalen Demokratiebegriff der poststrukturalistischen politischen Theorie. Dzudzek und Müller wenden sich gegen die Darstellung der riots als ,unpolitisch' und argumentieren, dass die Londoner Aufstände unbedingt politisch gewesen seien, und zwar vor allem deshalb, weil es ihnen gelungen sei, den ,normalisierten Ausnahmezustand' temporär aufzuheben und neue politische Subjekte zur Artikulation zu bringen. In den riots seien temporär neue politische Subjekte erschienen, die fundamentale Widersprüche der neoliberalen Gesellschaftsordnung aufgezeigt haben und schon deshalb unsere besondere Aufmerksamkeit verdienten.

Der Artikel „Planning in the Face of Democracy“ von Till Rosemann beschäftigt sich mit der Frage, ob institutionelle Raumplanung überhaupt demokratisch sein kann. Um dieses Thema zu diskutieren, führt Rosemann den radikalen Demokratiebegriff von Jacques Rancière ein und denkt ihn, so formuliert es der Autor, „mit dem? und in den Raum“. Dabei zeigt sich genau das paradoxe Feld der Differenz zwischen ,der Politik und ,dem Politischen'. Rosemann verdeutlicht, dass formelle staatliche Raumplanung der eigentlichen Sphäre des Politischen und dem Bereich des Demokratischen gar nicht angehören kann, da es bei einer solchen 
Planung immer darum geht, Ordnung herzustellen (und nicht darum, Ordnung aufzubrechen). Rosemann bleibt bei dieser Diagnose jedoch nicht stehen, sondern entwickelt einen Vorschlag, wie Raumplanung - ohne selbst im strengen Sinne demokratisch sein zu können - im Angesicht des Demokratischen und Politischen agieren kann und soll.

Juliane Karakayali und Birgit zur Nieden beschäftigen sich in ihrem Text „Rassismus und Klassen-Raum“ mit der aktuellen und historischen Segregation nach Herkunft an Berliner Grundschulen. Die Ausgangsbeobachtung der beiden Autorinnen ist, dass es an Grundschulen in vormals migrantisch geprägten Stadtteilen, in denen aktuell Gentrifizierungsprozesse stattfinden, verstärkt zu Segregationen nach Herkunft kommt. Diese zeigten sich in der Entstehung einzelner Schulen mit sehr hohem Anteil migrantischer Schüler_innen sowie einer Schulklasseneinteilung nach Herkunft. Der Artikel untersucht dieses Phänomen aus rassismustheoretischer Perspektive am Beispiel Berlin-Kreuzberg, wobei er aktuelle Segregationen im Kontext historischer Praktiken der getrennten Beschulung (den sogenannten Ausländerregelklassen) analysiert. Die Autor_innen verdeutlichen, dass sich im Sinne eines postliberalen Rassismus die Linien, entlang derer Trennungen vorgenommen werden, flexibilisieren und mit der gegenwärtigen Diskussion um „bildungsferne Kinder“ eine Legitimierung der rassistischen Diskriminierung stattfindet. Damit wird nicht zuletzt eine weitere Form des Postpolitischen selbst diskutiert.

In dem Beitrag „Die postdemokratische Stadt zwischen Politisierung und Kontinuität" gehen Daniel Mullis und Sebastian Schipper - ausgehend von einer kritischen Diskussion der konzeptionellen Zugänge bei Colin Crouch und Jacques Rancière - dem Gehalt der beiden Begriffsbestimmungen in der konkreten historischen Analyse nach, und zwar am Beispiel der Geschichte der kommunalen Selbstverwaltung in Frankfurt am Main. Entgegen der bei Crouch vorherrschenden Annahme, dass es vor der neoliberalen Stadt eine demokratische Form städtischen Regierens gegeben habe, wird unter Rückbezug auf die Argumentation Rancières zur Demokratie betont, dass der Fordismus keinesfalls als egalitärer, inklusiver oder demokratischer als der Neoliberalismus zu charakterisieren ist. Die fordistische Stadt, so die Autoren, sei zwar aus anderen Gründen, aber vom Grundsatz her nicht weniger postdemokratisch gewesen als die neoliberale Stadt der Gegenwart. Wirklich demokratische Momente würden sich allerdings in den Brüchen und Spalten der sozialen Konflikte der 1970er und 1980er finden.

„Occupy London und die besetzte Friern Barnet Library“ lautet der Titel des Artikels von Johannes Diesing, der von einer Hausbesetzung an einem eher ungewöhnlichen Ort berichtet, nämlich einer Bibliothek in einem bürgerlichen Stadtteil im Norden Londons. Diesing erzählt die Geschichte der Besetzung und kontextualisiert die daran anschließenden Aktionen der Occupy-Bewegung als Reaktion auf die neoliberalen städtischen Umstrukturierungen. Zudem verwendet Diesing die Postdemokratiethese von Jacques Rancière als Deutungsfolie der konkreten lokalen Praxis und auch der Schwierigkeiten, die mit diesem Vorgehen verbunden sind. Diesings Konfrontation seiner Feldstudie mit der politischen Theorie 
Rancières macht einen Vorschlag, wie es möglich ist, eine lokale politische Auseinandersetzung mit einer breiteren Kritik an einer neoliberalen Kürzungs- und Kommerzialisierungspolitik zu verknüpfen.

Catarina Gomes de Matos hat zu unserem Themenheft den Aufsatz „Das Modell Barcelona - Partizipation, Protest und Postpolitik“ beigetragen. Anlass ihrer Überlegungen ist, dass in dem gängigen Narrativ zur Stadtplanung in Barcelona die heutigen formellen Beteiligungsformen meist als Fortführung der nachbarschaftlichen Proteste in den 1970er Jahren gesehen werden. Die zunehmende Formalisierung von Bürger_innenbeteiligung gilt dabei gleichzeitig als zunehmende Demokratisierung. Gomes de Matos hinterfragt in ihrem Text den Zusammenhang von partizipativen Verfahren und Demokratie. Sie stellt dem traditionellen Demokratiebegriff ein emanzipatorisches Verständnis von Demokratie gegenüber, das sich an Theorien der poststrukturalistischen politischen Philosophie orientiert. Anhand der Darstellung des aktuellen Widerstands gegen Umbaumaßnahmen des Port Vell in Barcelona untersucht die Autorin, inwiefern formale Partizipationsmöglichkeiten die Teilhabe der Bevölkerung nicht nur nicht unterstützen, sondern letztlich einschränken oder ganz verhindern. Abschließend diskutiert Gomes de Matos, ob nicht formalisierte Proteste eine Alternative zu formellen Partizipationsverfahren darstellen könnten.

Wir freuen uns sehr, im Debattenteil den - für die zweite Ausgabe von $\mathrm{s} \mathbf{u} \mathbf{b} \backslash \mathbf{u} \mathbf{r} \mathbf{b}$ a $\mathbf{n}$ themengebenden - Aufsatz The Post-political City (2007) von Erik Swyngedouw in deutschsprachiger Übersetzung zu veröffentlichen. Swyngedouws Aufsatz ist aus unserer Sicht ein maßgeblicher Impuls zur Annäherung der neueren Theorien des Politischen an die kritische Stadtforschung und ein wichtiger Meilenstein für die in diesem Themenheft geführte Debatte. Unser im ersten Heft eingeführtes Debattenformat also der Abdruck eines Haupttextes mitsamt eingeladener Kritiken und Replik auf die Kritiken - werden wir in den kommenden Ausgaben von $\mathrm{s} \mathbf{u} \mathbf{b} \backslash \mathbf{u} \mathbf{r} \mathbf{b} \mathbf{a} \mathbf{n}$ weiter fortführen.

Im Magazin unseres Postpolitikheftes veröffentlichen wir einen Fotoessay, der sich unter dem konzeptionellen Rahmen der , ̈̈sthetik der Krise“ mit Street Art in Athen befasst. Julia Tulke geht in „Welcome to Athens" Spuren der sozialen Kämpfe und Proteste der letzten Jahre nach. Die Wände und Häuser der Stadt, so die Autorin, legten Zeugnis ab und erzählten „vom Alltag mit Krise und Austerität, von Angst, Verzweiflung, Repression, aber auch Hoffnung“.

Viel Spaß beim Lesen und Schauen wünscht die Redaktion Laura Calbet i Elias, Mélina Germes, Nina Gribat, Nelly Grotefendt, Jan Hutta, Boris Michel, Kristine Müller, Manfred Oberländer, Carsten Praum, Nikolai Roskamm, Nina Schuster 